\title{
Paeonol Ameliorates Chronic Itch and Spinal Astrocytic Activation via CXCR3 in an Experimental Dry Skin Model in Mice
}

\author{
Wen Wang ${ }^{1,2+}$, Qiaoyun $\mathrm{Li}^{1,2+}$, Zhongqiu Zhao ${ }^{3,4}$, Yutong Liu $^{5}$, Yi Wang ${ }^{5}$, Hui Xiong ${ }^{1,2 *}$ and \\ Zhinan $\mathrm{Mei}^{1,2 *}$
}

${ }^{1}$ School of Pharmaceutical Sciences, South-Central University for Nationalities, Wuhan, China, ${ }^{2}$ Institute of Ethnomedicine, South-Central University for Nationalities, Wuhan, China, ${ }^{3}$ Washington University School of Medicine, St. Louis, MO, United States, ${ }^{4}$ Barnes-Jewish Hospital, St. Louis, MO, United States, ${ }^{5}$ College of Life Sciences, South-Central University for Nationalities, Wuhan, China

\section{OPEN ACCESS}

Edited by:

Chi-Feng Hung,

Fu Jen Catholic University, Taiwan

Reviewed by:

Jia-You Fang,

Chang Gung University, Taiwan

Yu-Chun Huang,

Providence University, Taiwan

Chih-Chien Lin,

Providence University, Taiwan

${ }^{*}$ Correspondence:

Hui Xiong

xionghui0311020@163.com

Zhinan Mei

meizhinan@163.com

${ }^{\dagger}$ These authors have contributed equally to this work and share first

authorship

Specialty section:

This article was submitted to Ethnopharmacology,

a section of the journal

Frontiers in Pharmacology

Received: 30 October 2021

Accepted: 23 December 2021

Published: 13 January 2022

Citation:

Wang W, Li Q, Zhao Z, Liu Y, Wang Y, Xiong $H$ and Mei $Z$ (2022) Paeonol Ameliorates Chronic Itch and Spinal Astrocytic Activation via CXCR3 in an Experimental Dry Skin Model in Mice.

Front. Pharmacol. 12:805222.

doi: 10.3389/fphar.2021.805222
Paeonol is a bioactive phenol presents mainly in Paeonia suffruticosa Andr. (Paeoniaceae), Paeonia lactiflora Pall., and Dioscorea japonica Thunb. (Dioscoreaceae), harboring various pharmacological activities including anti-inflammatory, antioxidant, immune regulatory activity and reverse chemoresistance. Recent reports revealed paeonol exhibited good effects on chronic dermatitis, such as atopic dermatitis (AD) and psoriasis. However, whether paeonol is effective for dry skin disease and its mechanism of action still remain unclear. In this study, we analysed the effects of paeonol on a mouse model of dry skin treated with acetone-ether-water (AEW), which showed impressive activities in reducing scratching behavior and skin inflammation. To elucidate the underlying molecular targets for the anti-pruritic ability of paeonol, we screened the expression of possible chemokine pathways in the spinal cord. The expression of CXCR3 was significantly alleviated by paeonol, which increased greatly in the spinal neurons of AEW mice. In addition, treatment of paeonol significantly inhibited AEW-induced expression of astrocyte activity-dependent genes including TIr4, Lcn2 and Hspb1 et al. The inhibitory effects of paeonol on scratching behavior and astrocytic activation in the spinal cord induced by AEW were abolished when CXCR3 was antagonized or genetically ablated. Taken together, our results indicated that paeonol can ameliorate AEW-induced inflammatory response and itching behavior, and reduce the expression of spinal astrocyte activity-dependent genes induced by AEW, which are driven by CXCR3.

Keywords: paeonol, AEW, inflammation, anti-pruritic, CXCR3, astrocyte

\section{INTRODUCTION}

With the growth of aging population worldwide, age dependent diseases arouse broad public concern these years, including chronic dermatitis. Xerosis (often called dry skin) is one of the most common dermatological diagnosis in the elderly. The decreased content of natural moisturizing factors and lipids in the stratum corneum causes impaired enzymatic processes leading to the dry skin (WhiteChu and Reddy, 2011; Lichterfeld-Kottner et al., 2020). As the most common cause of pruritus in the elderly, dry skin induced repeated scratching in patients resulting in infections, ulcers and other 
serious complications (Moniaga et al., 2020). In this context, appropriate skin care strategies are effective to maintain and enhance the health and integrity of the elderly's skin (LichterfeldKottner et al., 2020).

Itch is a unique sensory experience, encoded by genetically distinguishable neurons in the peripheral nervous system (PNS) and central nervous system (CNS) (Dong and Dong, 2018). The itch signals are conveyed from the skin through primary afferents to the spinal dorsal horn (SDH), where it is processed and then sent to the brain via ascending pathways (Koga et al., 2020). Chronic itch has been widely recognized as an important clinical problem, but compared with other sensations (such as pain), its mechanism is poorly understood (Chen and Sun, 2020). Behavioral and pathophysiological evidence from Trpa1 ${ }^{-/-}$ mice indicated that the ion channel TRPA1 is required for both transduction of chronic itch signals to the CNS and for the dramatic skin changes triggered by dry-skin-evoked itch and scratching (Wilson et al., 2013; Moniaga et al., 2020). Recently, growing attention has been attracted to the central circuit mechanisms that contribute to itch sensation, including the crucial role of SDH astrocytes (Shiratori-Hayashi et al., 2015; Liu et al., 2016; Liu B. W. et al., 2019; Chen and Sun, 2020).

Chemokines are a family of small secreted proteins that bind to $G$ protein-coupled receptors to trigger intracellular signaling pathways and guide cell migration, proliferation, survival, and inflammation under homeostatic and pathological conditions. Mounting evidence supports the important roles of chemokine signaling in the peripheral and central nervous system in mediating chronic itch (Jiang et al., 2020). For example, the CCL2/CCR2, CXCL12/CXCR4, and CXCL10/CXCR3 signaling of small-diameter sensory neurons are involved in the pathophysiology of allergic contact dermatitis (ACD), eliciting itch- and pain-like behavior in SADBE murine model (Qu et al., 2015; Qu et al., 2017; Jing et al., 2018; Jiang et al., 2019; Su et al., 2020). Administration of a CXCR3 antagonist alleviated the itch behavior in dry skin model mice stimulated with acetone and diethyl ether followed by water (AEW), and $\mathrm{CxCr}^{-/-}$mice showed attenuated scratching in chronic itch models of dry skin and ACD (Qu et al., 2015; Qu et al., 2017; Jing et al., 2018). Moreover, AEW-induced activation of astrocytes in the spinal cord was suppressed in $C x \mathrm{Cr}^{-/-}$mice (Jing et al., 2018). Thus, targeting specific chemokines or chemokine receptors by natural compounds may provide novel therapeutic potential for the treatment of chronic itch.

Paeonol is a bioactive phenol mainly derived from Paeonia suffruticosa Andr. (Paeoniaceae), Paeonia lactiflora Pall., and Dioscorea japonica Thunb. (Dioscoreaceae) (Adki and Kulkarni, 2020), which have been applied for various diseases including fever, headache, cold, inflammation, skin diseases, neuralgia, allergy, rheumatoid arthritis, etc (Zhang et al., 2019). Since the first pharmacological activity of paeonol was reported in Traditional Chinese Medicine (TCM) 50 years ago (Harada and Yamashita, 1969), mounting evidence have reported the numerous pharmacologic effects of paeonol, such as antioxidant, anti-inflammatory, anti-cancer, apoptosis-inducing and neuroprotective activities (Jin et al., 2016; Liu et al., 2018; Gao et al., 2019; Zhang et al., 2020; Adki and Kulkarni, 2021).
Recently, paeonol has been exhibited as a potential therapeutic strategy for the treatment of allergic inflammatory conditions in psoriasis and atopic dermatitis (AD) (Meng et al., 2017; Meng et al., 2019), but whether paeonol is effective in the treatment of dry skin? And if yes, what is the possible mechanism? In order to answer these questions, we carried out the experimental work of this study.

\section{MATERIALS AND METHODS}

\section{Reagents}

Paeonol (Cat\# 552-41-0, $\geq 98 \%$ purity) was purchased from Sichuan Weikeqi Biological Technology Co., Ltd (Sichuan, China) and made into 5\% paeonol ointment (the approximate final volumetric molar concentration of paeonol is $300 \mathrm{mM}$ ) with other ingredients consist of stearic acid, potassium carbonate, glyceryl monostearate, triethanolamine, glycerin and appropriate amount of water. The positive control drug TRPA1 antagonist HC-030031 (Cat\# HY-15064, $\geq 95.91 \%$ purity) and CXCR3 antagonist AMG487 (Cat\# HY-15319, $\geq 99.51 \%$ purity) were purchased from MedChemExpress (United States).

\section{Animals}

Male C57BL/6J mice of 7 weeks $( \pm 20 \mathrm{~g}$ ) were purchased from Liaoning Changsheng Biotechnology Co., Ltd (Changchun, China). $\mathrm{Cxcr}^{-/-}$mice (Jing et al., 2018) were provided by Dr. Yongjing Gao at Nantong University in China. All mice were kept in the condition of $22 \pm 2^{\circ} \mathrm{C}$ with $55 \pm 15 \%$ humidity under a $12 \mathrm{~h}$ light/dark cycle and free access to food and water in air-filtered cages. All animal procedures were performed in accordance with the Guidelines for Care and Use of Laboratory Animals of SouthCentral University for Nationalities (SYXK 2016-0089), and were approved by the Animal Ethics Committee of South-Central University for Nationalities (2017-SCUEC-ACE-023).

\section{Mouse Model of AEW and Treatment}

The AEW model was chosen for in vivo pharmacological study of dry skin and established as previously described (Wilson et al., 2013; Zhao et al., 2013; Moniaga et al., 2020). Briefly, the nape of mice was shaved 2 days in advance. Dry skin was induced twice a day for six consecutive days by application of a $1.5 \mathrm{ml} \mathrm{1:1} \mathrm{mixture} \mathrm{of} \mathrm{acetone} \mathrm{and} \mathrm{diethyl}$ ether for $20 \mathrm{~s}$, followed by clean water for $30 \mathrm{~s}$, and the animals of blank group were treated with water only. All AEW-induced mice were randomly divided as follows $(n=$ 6-7 each group): Model group (applied with Blank matix ointment, referred to as the "AEW" group), low-dose group (applied with Pae ointment, $50 \mathrm{mg} / \mathrm{kg}$, referred to as "Pae-L" group), middle-dose group (applied with Pae ointment, $100 \mathrm{mg} / \mathrm{kg}$, referred to as "Pae-M" group), high-dose group (apply with Pae ointment, $150 \mathrm{mg} / \mathrm{kg}$, referred to as "Pae-H" group), and positive control group (intraperitoneal injection with TRPA1 inhibitor HC-030031 (Fernandes et al., 2013; Oh et al., 2013; Patricio et al., 2015; Wilzopolski et al., 2021), $10 \mathrm{mg} / \mathrm{kg}$, referred to as "PC" group). We refer to previous studies to determine the in vivo dose of paeonol (Xue et al., 
TABLE 1 | Oligo nucleotide sequences of the primers used for qPCR.

\begin{tabular}{|c|c|c|}
\hline Gene & Forward $\left(5^{\prime}\right.$ to $\left.3^{\prime}\right)$ & Reverse (5' to $\left.3^{\prime}\right)$ \\
\hline Actin & GTACTCTGTGTGGATCGGTGG & AAACGCAGCTCAGTAACAGTCC \\
\hline $\mathrm{Cc} / 1$ & GCATGCTTACGGTCTCCAATAG & GCAGGGGTTCACCTTCTTCA \\
\hline Ccl2 & GATGCAGTTAACGCCCCACT & ACCCATTCCTTCTTGGGGTC \\
\hline Ccl4 & GCTGTTCTCTTACACCTCCCG & CAGTTCAACTCCAAGTCACTCATGT \\
\hline Ccl5 & TGCCCACGTCAAGGAGTATाT & GATGTATTCTTGAACCCACTTCTTC \\
\hline Ccl6 & TATCCTTGTGGCTGTCCTTGG & TGATAAAGATGATGCCCGGCT \\
\hline Ccl7 & TTCTGTGCCTGCTGCTCATAG & СTTCCATGCCCTTCTITGTCT \\
\hline Ccl8 & CTGCTCATAGCTGTCCСTGTCA & CACTGGATATTGTTGATTCTCTCGT \\
\hline Cc/9 & TGGGTCTGCCCACTAAGAAG & CCCTTGCTGTGCCTTCAGAC \\
\hline Ccl11 & СTGCTCACGGTCACTTCCTTC & GAAGACTATGGCTICAGGGTGC \\
\hline Ccl12 & GCTACCACCATCAGTCCTCAGG & TGGCTGCTTGTGATTCTCCTGT \\
\hline Ccl17 & CGAGAGTGCTGCCTGGATTAC & CCCTGGACAGTCAGAAACACG \\
\hline Ccl20 & CCTTCCAGAGCTATTGTGGGTT & СTCTTAGGCTGAGGAGGTTCAC \\
\hline $\mathrm{Ccl} 2$ & СTCAAAATCCTGCCGCAAGC & AGGTGAGTAAAGGTGGCGTC \\
\hline Cxcl1 & CCAAACCGAAGTCATAGCCA & TGGGGACACCTITAGCATCT \\
\hline $\mathrm{Cxcl} 2$ & GCTGTCCCTCAACGGAAGAA & CAGGTACGATCCAGGCTTCC \\
\hline Cxcl5 & CACTCGCAGTGGAAAGAACG & CGTGGGTGGAGAGAATCAGC \\
\hline Cxc/9 & TCСССТАAАTCTTCCACAGTGC & AGGCCAAAGGTTAGTTAGGCAA \\
\hline Cxc/10 & AАATCATCCCTGCGAGCCTATC & CTAGCCATCCACTGGGTAAAGG \\
\hline $\mathrm{CxC} / 11$ & GGCTTCCTTATGTTCAAACAGGG & CACTTCAACTITGTCGCAGCC \\
\hline $\mathrm{Cxc} / 13$ & TATGTGTGAATCCTCGTGCCAA & GCTTCAGGCAGCTCTTCTCTTA \\
\hline Cor1 & AGGTTGGGACCTTGAACCTT & TGGAGTGGAGTCCCCATAGT \\
\hline Ccr2 & AGGAGCCATACCTGTAAATGC & TAGTCATACGGTGTGGTGGC \\
\hline Ccr3 & AACTTGCAAAACCTGAGAAGC & GGGTGGTGCCCACTCATATT \\
\hline Ccr5 & AGCCGGGAAGGTAGTCTCAT & AGTCCCGGTGTGGTAGGATT \\
\hline Ccr6 & CTGGGCAGTTACTCATGCCA & CACTGCCACACAGATGACCT \\
\hline Ccr7 & GGCCAACTTCAACATCACCAAT & GCATACAAGAAAGGGTTGACGC \\
\hline Ccr8 & GTGGGCAGCTCTGAAACCTC & GAGGAGGAACTCTGCGTCAC \\
\hline Ccr9 & GCAGGCTGTTGACGCTTATG & CCTTCGGAATCTCTCGCCAA \\
\hline Cor10 & ATGTCCAGGCTITCAGTCGG & GAGGTGGGAGATCGGGTAGT \\
\hline Cxcr2 & GAGCCACTCTGCTCACAAAC & AGCAGAGTCACCAGGACGTA \\
\hline Cxcr3 & AGCCATGTACCTTGAGGTTAGT & AGGTTCTGTCAAAGTTCAGGCT \\
\hline Cxcr4 & TGGAACCGATCAGTGTGAGT & TGTCCGTCATGCTCCTTAGC \\
\hline Cxcr5 & CAGCACAAACCTTCTCGACATC & GCTGTTACTGTAGAAGGCCAGT \\
\hline CX3cr1 & CACTTGCCTCTGGTGGAGTC & GGAAGGAGGTGGACATGGTG \\
\hline TIr4 & TGGCTGGTTTACACGTCCAT & TGCAGAAACATTCGCCAAGC \\
\hline Lcn2 & TGAGTGTCATGTGTCTGGGC & GAACTGATCGCTCCGGAAGT \\
\hline Hspb1 & ATCACTGGCAAGCACGAAGA & GGCCTCGAAAGTAACCGGAA \\
\hline $\mathrm{C} d 44$ & ССTCTGCCAGGCTITCAACA & TGCACAGATAGCGTTGGGAT \\
\hline$C p$ & GGTCCTGTCATITGGGCAGA & CATGGGAGGCTTGCTGTGA \\
\hline Serpina3n & TGGCCTCCATCAACACTGAC & AAAGCCCTGGTGGATGTCTG \\
\hline Vim & GCCAGCAGTATGAAAGCGTG & ACCTGTCTCCGGTACTCGTT \\
\hline Gfap & GGCGAAGAAAACCGCATCAC & GGTGAGCCTGTATTGGGACAA \\
\hline
\end{tabular}

2017; Xiao et al., 2020). The drugs were administrated once daily at 12:00 AM from day 4 to day 6 . On day 7 , the spontaneous scratching behavior of mice was recorded for $1.5 \mathrm{~h}$. For behavioral test using CXCR3 antagonist, mice were pre-treated with AMG487 (5 mg/kg) $30 \mathrm{~min}$ before paeonol application $(150 \mathrm{mg} / \mathrm{kg})$ on day 7 ( $n=7-8$ mice per group). The hind paw scratching behaviors were recorded for $60 \mathrm{~min}$ and analyzed by people blinded to the experimental design, which is defined as a lifting of the hind limb towards the shaved area at the back of the neck and then a replacing of the limb back to the floor (Sun et al., 2009; Wang et al., 2020).

\section{Pathomorphological Study of Skin}

The nape skin of mice was collected and fixed in $4 \%$ paraformaldehyde (PFA), routinely dehydrated, embedded in paraffin, and sectioned. Haematoxylin and eosin (H\&E) and toluidine blue staining were performed as previously described (Zhao et al., 2013; Xiang et al., 2020). After the stained sections were dehydrated, cleared and sealed, they were visualized under a microscope for photograph. ImageJ software was used to measure the thickness of epidermis and count the number of mast cells of dermis $(n=3$ mice per group).

\section{ELISA Assay}

Protein levels of IL-1 $\beta$, IL- 4 and IL-6 in serum were measured using ELISA kits (Bio-Swamp Life Science, Wuhan, China) according to the manufacturer's protocol ( $n=4-8$ each group). The absorbance was measured at $450 \mathrm{~nm}$ using a microplate reader (Thermo Scientific, Juensuu, Finland) (Wang et al., 2020). 

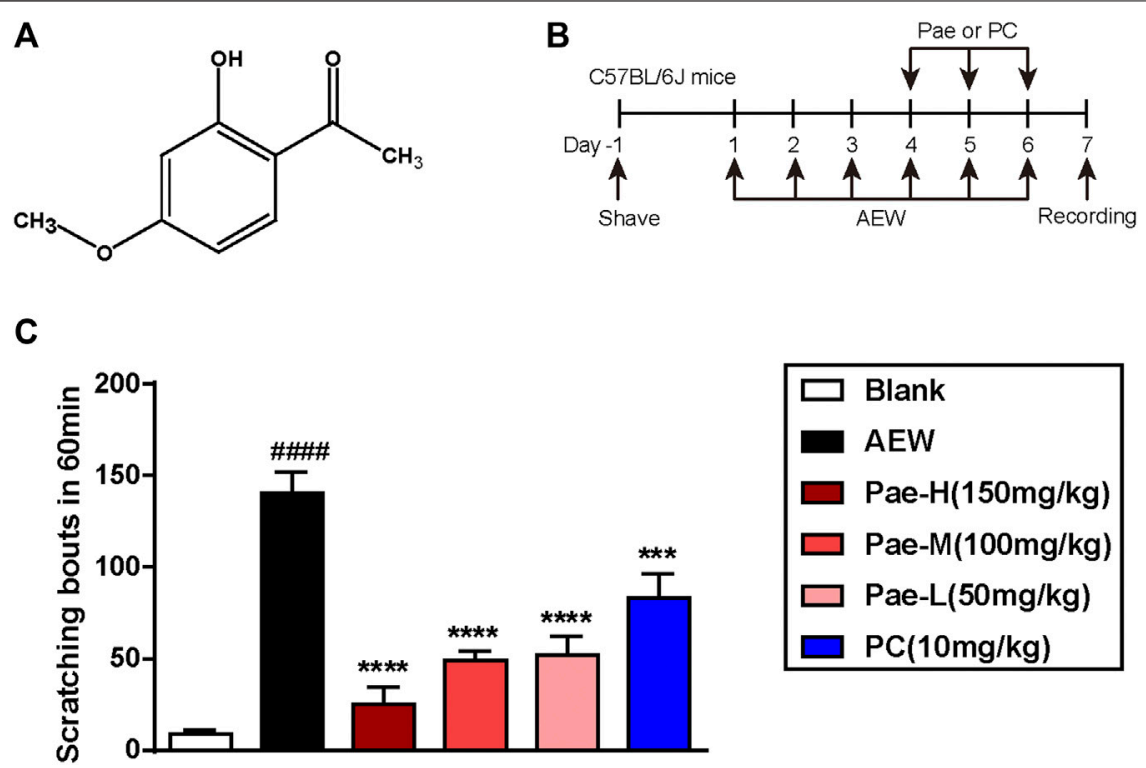

FIGURE 1 | Paeonol alleviated AEW-induced scratching behavior of dry skin mice. (A) The chemical structure of paeonol. (B) Schematic diagram of the animal experimental protocol. Spontaneous scratching of mice was videotaped and analysed. (C) The behavior results of different groups on day 7 . Data were expressed as mean \pm SEM. ${ }^{\# \# \#} p<0.001$, compared with the blank group. ${ }^{* *} p<0.01$, ${ }^{* \star *} p<0.001$, compared with the AEW model ( $n=6-7$ each group). Pae-H, paeonol-high dose; Pae-M, paeonol-middle dose; Pae-L, paeonol-low dose; PC, positive control drug, HC-030031. The relevant labels in the following figures are the same unless otherwise mentioned.

\section{Real-Time Quantitative PCR}

RT-qPCR was performed as previously described (Feng et al., 2017). Briefly, total RNA of spinal cord was extracted using RNAiso Plus (Takara) and reverse transcribed using a Revert Aid First-Strand cDNA Synthesis kit (Thermo) according to the manufacturer's instructions. The cDNA was subjected to RTqPCR with corresponding primer sets using SYBR Green Master Mix (Vazyme Biotech Co. Ltd., Nanjing, China) (Liu S. et al., 2019; Wang et al., 2020), and the reaction was hot-started at $95^{\circ} \mathrm{C}$ for $2 \mathrm{~min}$ and then incubated at $95^{\circ} \mathrm{C}$ for $15 \mathrm{~s}, 60^{\circ} \mathrm{C}$ for $30 \mathrm{~s}$, and $72^{\circ} \mathrm{C}$ for $1 \mathrm{~min}$ for 40 cycles. The primer sequences of targeted genes are listed in Table 1.

\section{Western Blotting}

The cervical spinal cord of mice was homogenized in the ice-cold RIPA lysis buffer (Bimake, China) containing protease inhibitors (Bimake, China) and phosphatase inhibitors (Roche, Germany) (Xu et al., 2021), lysed for $30 \mathrm{~min}$, and then centrifuged at $12,000 \mathrm{rpm} 4^{\circ} \mathrm{C}$ for $10 \mathrm{~min}$. Next, the supernatant was mixed with $5 \times$ SDS loading buffer and incubated at $95^{\circ} \mathrm{C}$ for $10 \mathrm{~min}$. Then, the boiled protein lysate was separated by $10 \%$ SDS-PAGE and transferred to $0.22 \mu \mathrm{m}$ PVDF membranes. After blocking with $5 \% \mathrm{BSA}$ for $2 \mathrm{~h}$ at room temperature (RT), the membranes were incubated with primary antibodies at $4^{\circ} \mathrm{C}$ overnight, washed in TBST, and incubated with appropriate HRP-conjugated secondary antibodies for $2 \mathrm{~h}$ at RT. The protein bands were visualized by using BeyoECL Plus (P0018, Beyotime, China), and a densitometry analysis was performed on an imaging system (Tanon Science and Technology Co., Ltd., Shanghai, China) (Liu S. et al., 2019). The primary antibodies used in this study are antiCXCR3 (26756-1-AP, Proteintech, 1:1,000) and anti-GAPDH (\#6176106, CST, 1:10,000).

\section{Immunofluorescence Staining}

Mice was perfused with 4\% PFA and the cervical spinal cord was dissected. Next, the tissues were fixed in $4 \% \mathrm{PFA}$ overnight at $4^{\circ} \mathrm{C}$, followed by processing in $20 \%$ sucrose, embedded in OCT, and then sectioned into $20 \mu \mathrm{m}$, and subjected to tissue staining after cryosection. A blocking solution was prepared with $0.1 \%$ Tritox$100,3 \%$ goat serum, $0.1 \%$ BSA plus PBS solution, and blocked at room temperature for $1 \mathrm{~h}$ (Xiang et al., 2020). After blocking, sections were incubated overnight at RT with the specific primary antibodies (anti-CXCR3, 1:5,000, 26756-1-AP, Proteintech; antiNeuN, 1:200, Millipore, MAB377; anti-GFAP, 1:500, Millipore, MAB360; anti-CD11b, 1:200, Abcam, ab8878). Then, they were washed 3 times in PBS, and incubated with the appropriate secondary antibodies (Alex Fluor ${ }^{\mathrm{TM}} 647$ goat anti-rabbit IgG $(\mathrm{H}+\mathrm{L}), 1: 1,000$, Thermo Fisher Scientific, \#1851447; Alex Fluor $^{\mathrm{TM}} 568$ goat anti-mouse $\operatorname{IgG}(\mathrm{H}+\mathrm{L}), 1: 300$, Thermo Fisher Scientific, 1862187) for $1 \mathrm{~h}$ at RT. After incubated with $5 \mu \mathrm{g}$ DAPI, tissue sections were washed, mounted and then imaged after drying. All imaging were performed on a Zeiss Axio Observer 7 Fluorescence Microscope. The immune signal intensity was quantified in a semi-automatic manner using 


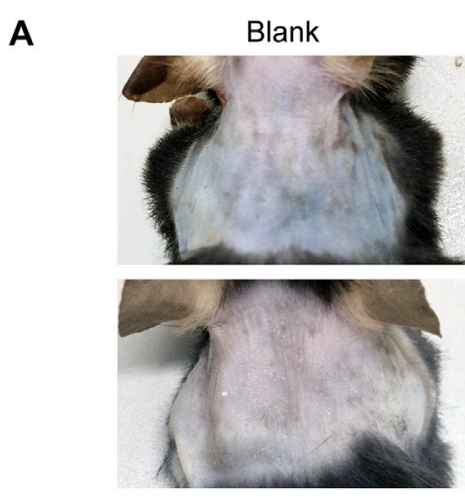

Pae-H

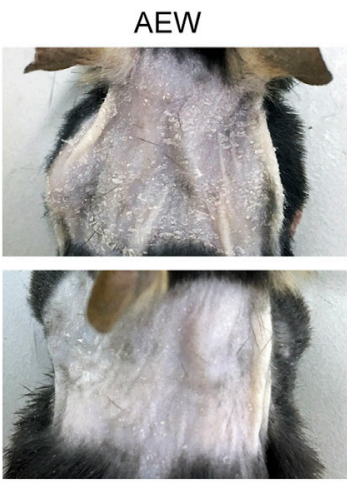

Pae-M

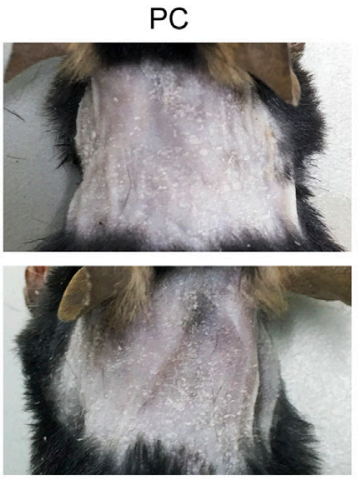

Pae-L
B

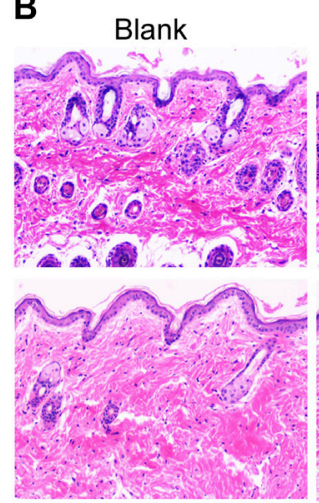

Pae-H
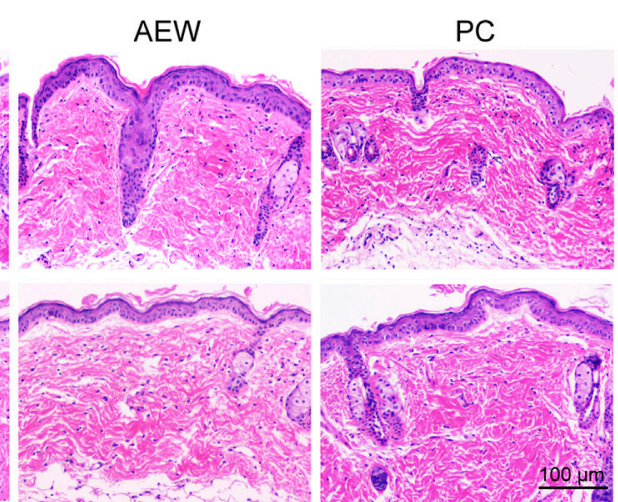

Pae-L
Pae-M

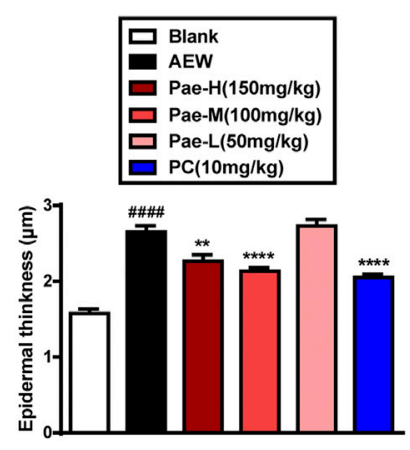

C

Blank

AEW

PC

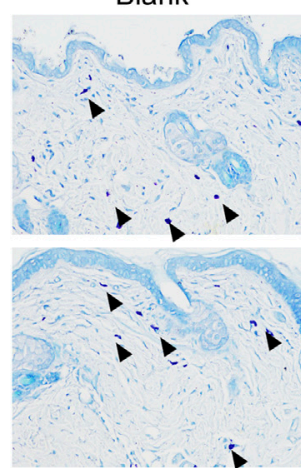

Pae-H

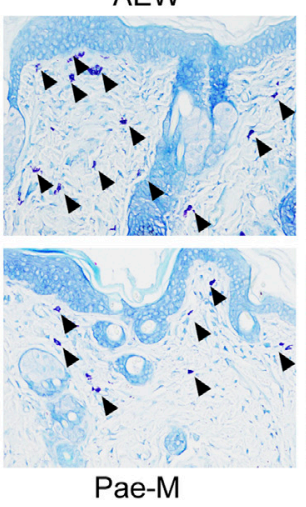

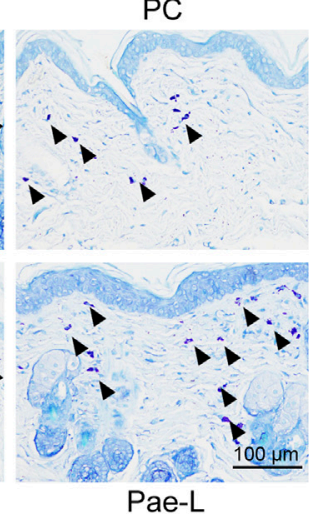

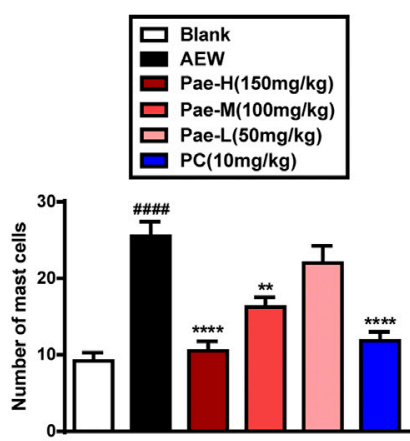

Pae-L

FIGURE 2|Paeonol improved the pathological changes of dorsal neck skin in AEW mice. (A) The representative images of dorsal neck skin of each group on day 7.

(B) The representative images of Haematoxylin and eosin (H\&E) staining and the thickness of epidermis were measured. (C) The representative images of toluidine blue

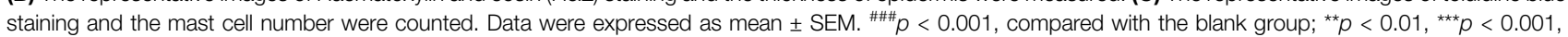
compared with the AEW model group ( $n=3$ each group). 

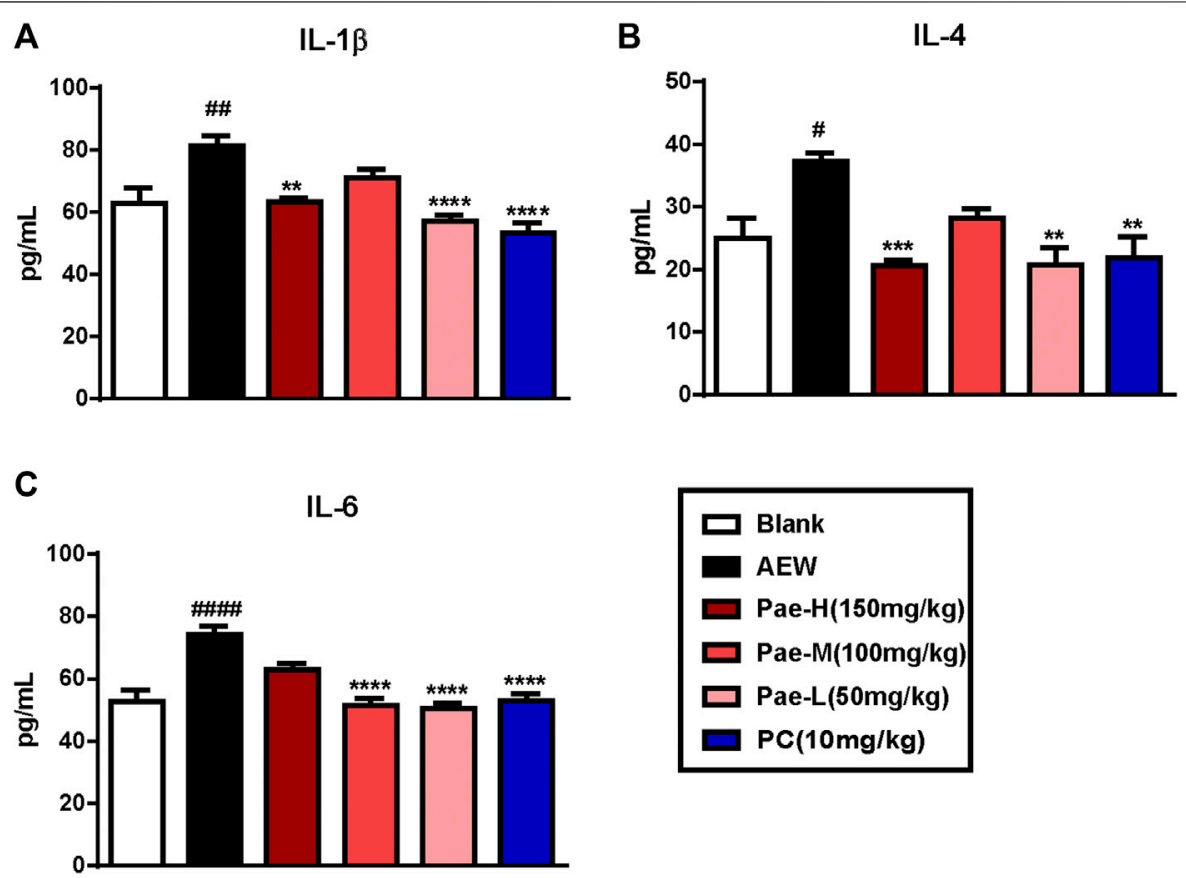

FIGURE 3|Paeonol reduced protein levels of pro-inflammatory cytokines in the serum of AEW mice. (A-C) Administration of paeonol decreased the levels of IL-1 $\beta$ (A), IL-4 (B), IL-6 (C) in the serum of AEW-induced mice. Data were expressed as mean \pm SEM. ${ }^{\#} p<0.05$, ${ }^{\# \#} p<0.01$, ${ }^{\# \# \# ~} p<0.0001$, compared to the blank group; ${ }^{* *} p<0.01,{ }^{* * *} p<0.001,{ }^{* * *} p<0.0001$, compared to the AEW model group ( $n=4-7$ each group).

Image-Pro Plus software and 4-5 images were counted for each mouse as described previously (Zhao et al., 2013; Xiang et al., 2020).

\section{Statistical Analysis}

All data are presented as the mean \pm SEM. GraphPad Prism five software (San Diego, California, United States) was used for the statistical analysis of the data and generation of the graphics. Student's $\mathrm{t}$ test was used to analyse statistical comparisons between two groups. One-way analysis of variance (ANOVA) followed by Bonferroni's post hoc test was used for multiple group comparisons (Liu S. et al., 2019). $p$ value $<0.05$ was considered statistically significant.

\section{RESULTS}

\section{Paeonol Attenuated the Scratching Behavior in AEW-Induced Mice}

Paeonol (2-hydroxy-4-methoxyacetophenone) is a bioactive phenol (Figure 1A) which has various pharmacological potential for clinical therapeutics (Jin et al., 2016; Meng et al., 2017; Liu et al., 2018; Gao et al., 2019; Meng et al., 2019; Adki and Kulkarni, 2020). To access the effect of paeonol against dry skin, mice were applied with AEW on the shaved nape twice daily for 6 days, and paeonol or positive control (PC) drug were administrated for 3 days starting from day 4 (Figure 1B). Since TRPA1 is required for both transduction of chronic itch signals to the CNS and for the dramatic skin changes triggered by dry-skin-evoked itch and scratching (Wilson et al., 2013; Feng et al., 2017), we chose its antagonist HC-030031 as the PC. Consistently, mice stimulated with AEW exerted intense spontaneous scratching on day 7 (Figure 1C), and three doses of paeonol significantly reduced the scratching bouts in a dose-dependent manner. It's worth noting that paeonol exhibited a trend to be superior to PC in alleviating the chronic itch induced by AEW.

\section{Paeonol Improved the Pathologic Changes of Skin in Mice Induced by AEW}

As described in previous studies, the mice neck stimulated with AEW showed severe skin lesions characterized by a dry, scaly and rough surface (Zhao et al., 2013). Administration of paeonol $(150 \mathrm{mg} / \mathrm{kg}, 100 \mathrm{mg} / \mathrm{kg})$ efficiently improved these pathologic changes, while the skin appearance of PC group showed no significant difference with that of model mice (Figure 2A). To further explore the anti-inflammatory effect of paeonol, pathological analysis of skin slices were conducted. Results of H\&E (Figure 2B) and toluidine blue (Figure 2C) staining indicated that paeonol $(150 \mathrm{mg} / \mathrm{kg}, 100 \mathrm{mg} / \mathrm{kg})$ showed good 

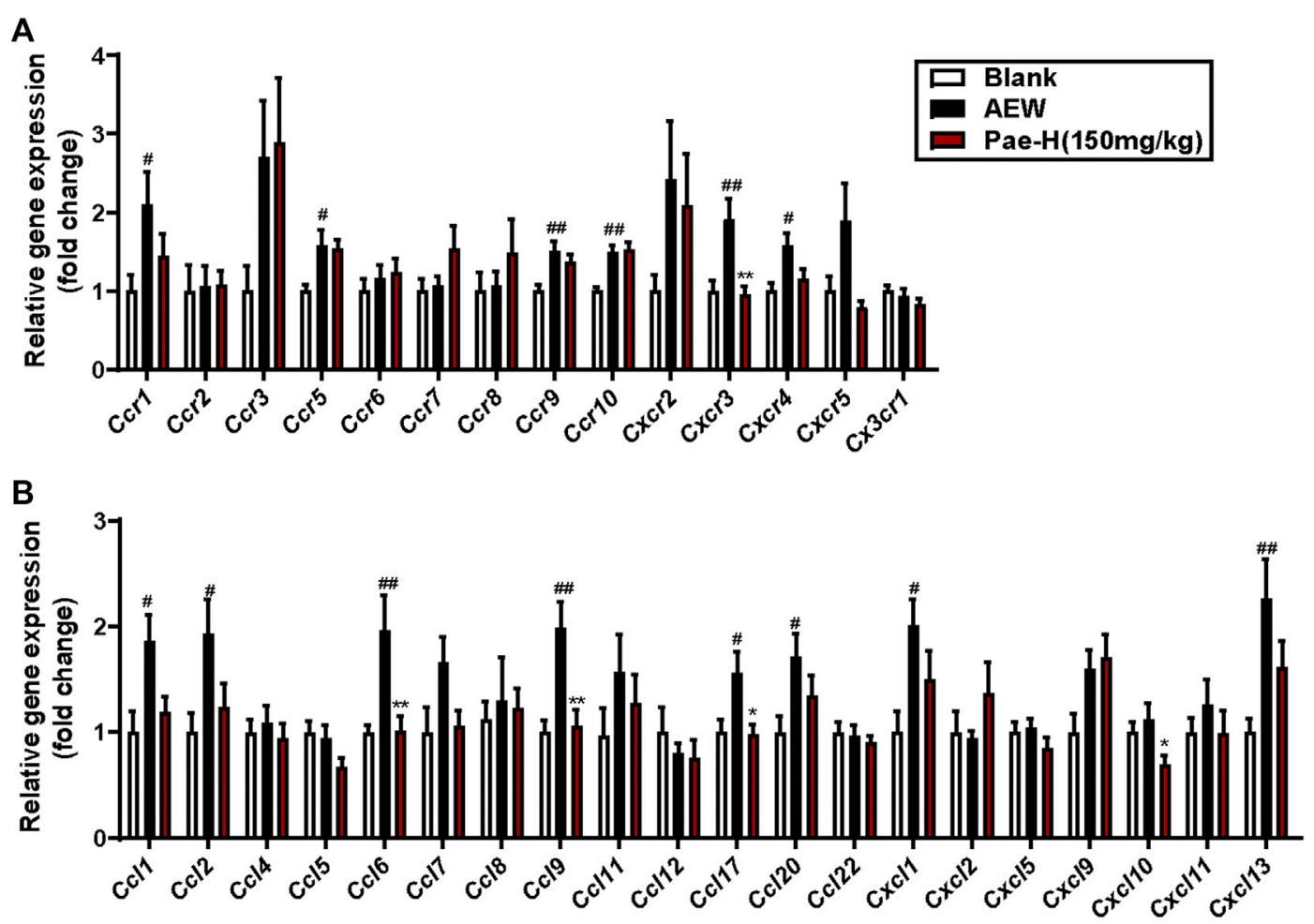

FIGURE 4 | The effects of paeonol on the expression of chemokine receptors and ligands in the spinal cords. The increased mRNA levels of chemokine receptor Cxcr3 (A) and chemokines including Cc/6, Cc/9, and C C/17 (B) in the spinal cord of AEW mice were decreased by paeonol application. Data were expressed as mean \pm SEM. ${ }^{\#} p<0.05,{ }^{\# \#} p<0.01,{ }^{\# \# \#} p<0.001$, compared to the blank group; ${ }^{*} p<0.05,{ }^{* * *} p<0.01$, compared to the AEW model group ( $n=4-8$ each group).

effects on improving the corneous hyperplasia and mast cell infiltration induced by AEW, which was similar to PC drug.

\section{Paeonol Decreased the Pro-Inflammatory Cytokine Levels in Serum of AEW Mice}

Alterations in the protein levels of cytokines IL1- $\beta$, IL-4 and IL-6 were analyzed by ELISA in order to illustrate the antiinflammatory effects of paeonol. The expression of IL-1 $\beta$, IL-4 and IL- 6 were increased in the serum of AEW group compared to the blank group (Figures $\mathbf{3 A}-\mathbf{C}$ ). On the other hand, treatment with paeonol inhibited AEW-induced cytokine expression, and the protein levels of IL- $1 \beta$, IL- 4 and IL- 6 were significantly lessened in paeonol-treated mice.

\section{Paeonol Reduced the Upregulation of CXCR3 in the Spinal Cord of AEW Mice}

The chemokine signalling of CNS plays a vital role in mediating chronic itch (Jing et al., 2018; Jiang et al., 2020). Thus, we used RT-qPCR to screen the expression of spinal chemokine pathways to explore the potential mechanism for paeonol's anti-pruritic activity. Among chemokine receptors (Figure 4A) and chemokine ligands (Figure 4B) increased in the spinal cord of AEW group, paeonol significantly reduced the expression of CXCR3. Besides, paeonol also reduced the mRNA level of CXCL10, which is the one of the ligands of CXCR3. Emerging evidence proposed that CXCR3/CXCL10 signaling in the spinal cord may mediate central sensitization underlying chronic itch and alloknesis in AEW model (Jing et al., 2018). Therefore, we proposed that paeonol may exhibited an anti-pruritic effect via suppressing CXCR3 in the spinal cord.

\section{The Expression Profile of Chemokine Receptor CXCR3 in the Spinal Cord of AEW Mice}

To further verify the contribution of CXCR3 to AEW-induced pruritus of mice, we examined the expression of spinal CXCR3 at a series of time points. Results of RT-qPCR and western blotting demonstrated that both mRNA and protein levels of CXCR3 gradually elevated in the spinal cord of AEW mice (Figures $\mathbf{5 A}, \mathbf{B})$, suggesting that CXCR3 was likely involved in the pathogenesis of dry skin. Then, the expression profile of 

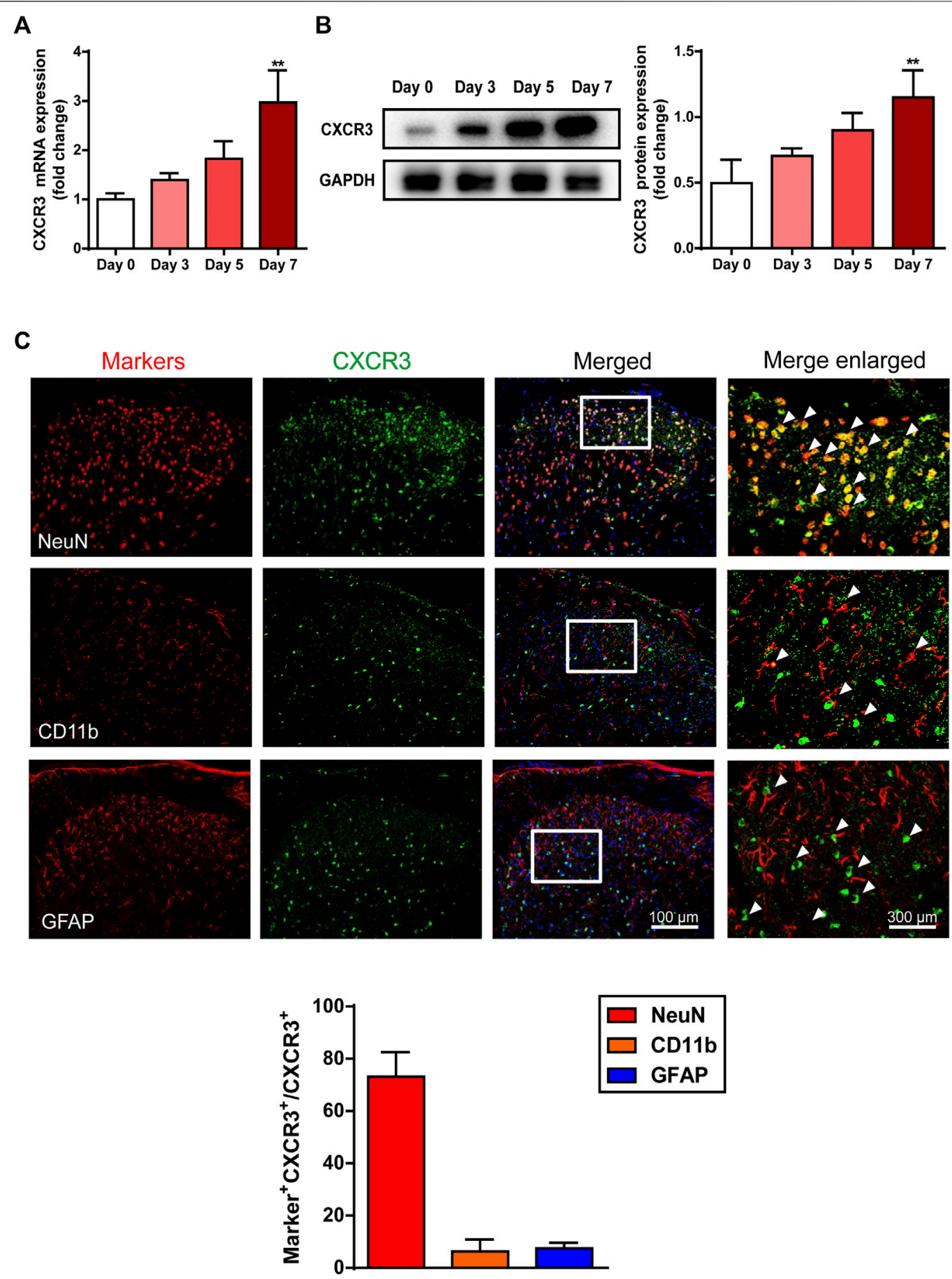

FIGURE 5 | The expression profile of CXCR3 in the spinal cord in the AEW-stimulated mice. The mRNA (A) and protein (B) level of CXCR3 in the spinal cord increased as stimulation of AEW went on ( $n=3-7$ each group). (C) Immunofluorescence co-staining images of CXCR3 with neuron marker (NeuN), microglial marker (CD11b) and astrocyte marker (GFAP) in the spinal dorsal horn of AEW mice ( $n=4$ each group). Data were expressed as mean \pm SEM. ${ }^{* *} p<0.01$, compared to the Day 0 group.

CXCR3 in the spinal dorsal horn was investigated by immunofluorescence (IF) assay. Double-staining of CXCR3 with NeuN, CD11b and GFAP respectively showed that
CXCR3 dominantly located in $\mathrm{NeuN}^{+}$neurons, but not $\mathrm{CD} 1 \mathrm{~b}^{+}$microglia cells or $\mathrm{GFAP}^{+}$astrocytes (Figure $5 \mathrm{C}$ ) in the spinal dorsal horn. 


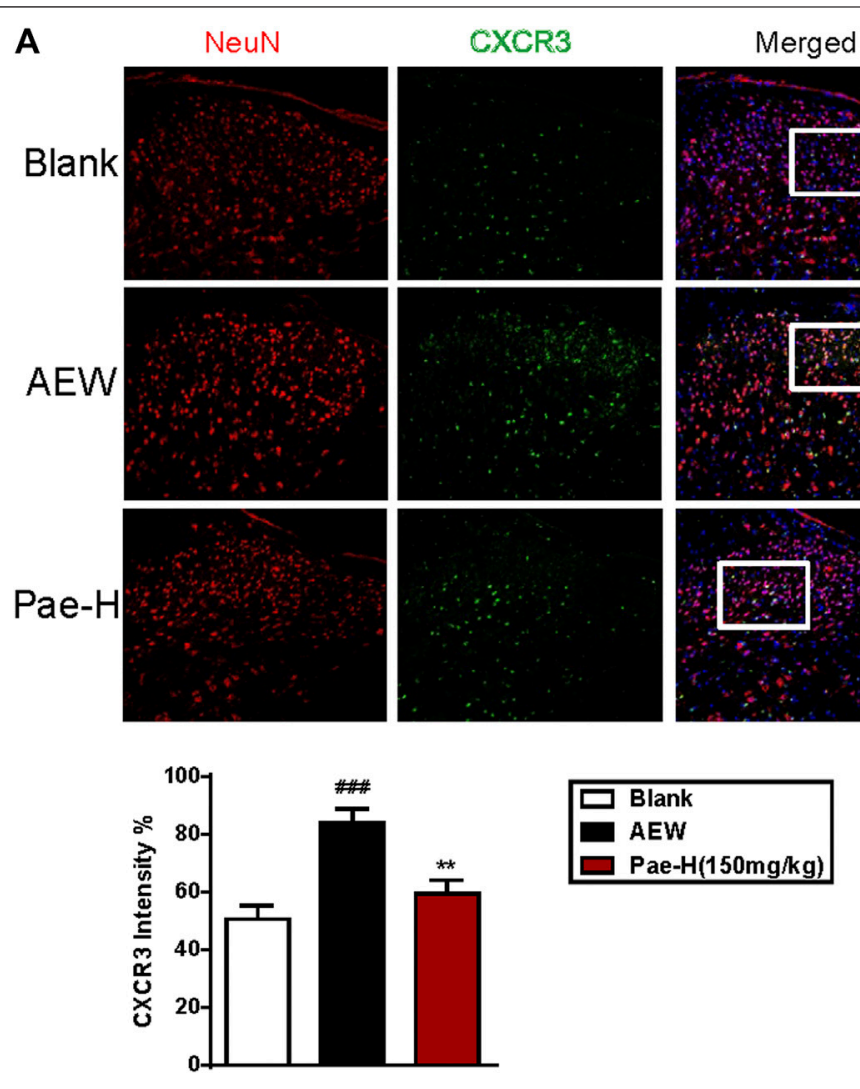

B
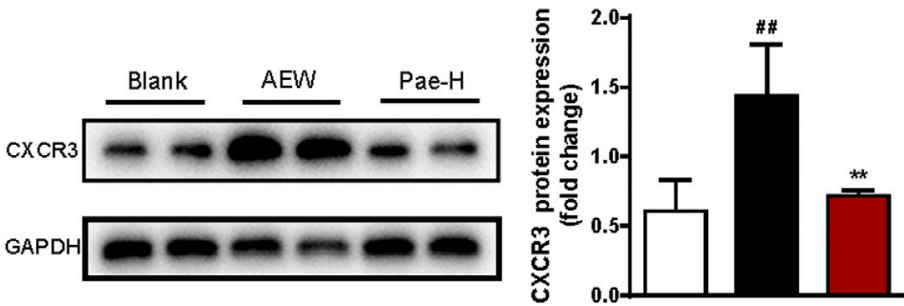

FIGURE 6 | Paeonol decreased the expression of CXCR3 in the spinal neurons of AEW group. (A) The results of immunofluorescence indicated paeonol downregulated the $\mathrm{CXCR}^{+} \mathrm{NeuN}^{+}$double-positive cell ratio in the AEW spinal cord ( $n=4$ each group). (B) The results of western blot demonstrated paeonol reduced the protein level of spinal CXCR3 in AEW model group $\left(n=4\right.$ each group). Data were expressed as mean \pm SEM. ${ }^{\# \#} p<0.01$, ${ }^{\# \#} p<0.001$, compared to the blank group; ${ }^{* *} p<0.01$, compared to the AEW model group.

\section{Paeonol Inhibited the Expression of Neuronal CXCR3 in the Spinal Cord of AEW Mice}

Next, we analyzed the effects of paeonol on CXCR3 expression of spinal neurons by IF staining. As shown in Figure 6A, AEW stimulation dramatically raised the ratio of $\mathrm{CXCR}^{+} \mathrm{NeuN}^{+}$double positive cells in the spinal dorsal horn, and application of paeonol efficiently reversed the increased intensity of CXCR3 in $\mathrm{NeuN}^{+}$ neurons. Results of western blotting also supported this consequence (Figure 6B), as AEW-induced upregulation of spinal CXCR3 was significantly decreased by paeonol administration.

\section{Paeonol Suppressed AEW-Induced Chronic Itch in a CXCR3-Dependent Way}

Then, we observed the effects of paeonol on the scratching bouts of AEW mice pre-treated with a single intraperitoneal injection of CXCR3 antagonist AMG487. The data of itching behavior 

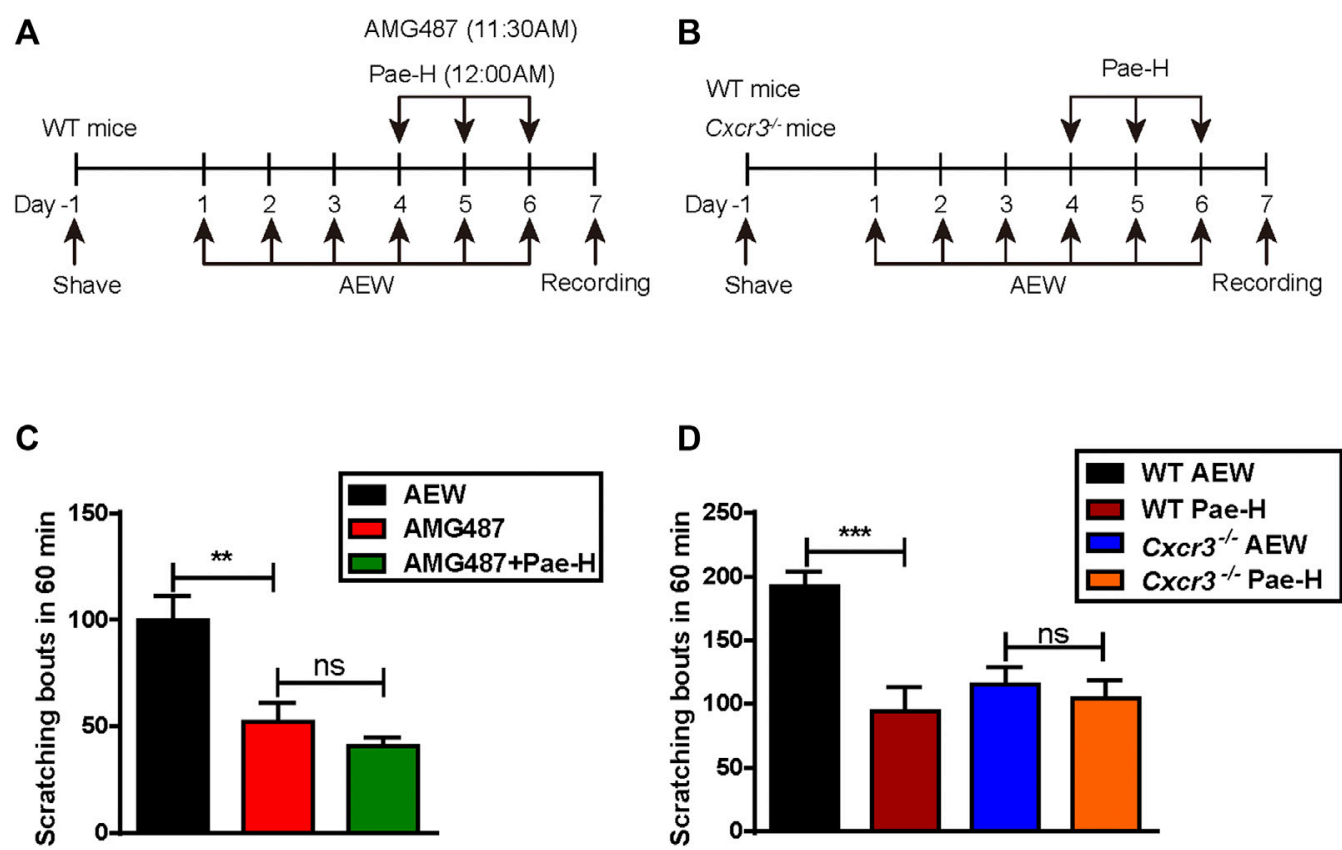

FIGURE 7 | The effects of paeonol on chronic itch in AMG487 pre-treated WT and CXcr3 ${ }^{-/-}$mice. (A) Schematic protocol of CXCR3 inhibition and induction of AEW-induced dry skin model. (B) Schematic protocol of AEW-induced dry skin model in $\mathrm{Cxcr}^{-/-}$mice. (C) The effects of paeonol on AEW-induced spontaneous scratching in WT mice pre-treated with AMG487. (D) The effects of paeonol on AEW-induced spontaneous scratching in $C \times C r 3^{-/-}$mice $(n=7-9$ each group). Data were expressed as mean \pm SEM. ${ }^{* *} p<0.01,{ }^{* * * *} p<0.001$, compared to the WT-AEW model group.

indicated that paeonol failed to relieve the AEW-stimulated itch when CXCR3 was pharmacologically inhibited (Figures 7A-C). Similarly, though paeonol significantly impaired the scratching frequency in $\mathrm{AEW}$-induced $\mathrm{C} 57 \mathrm{BL} / 6 \mathrm{~J}$ mice, it was almost ineffective in $\mathrm{CxCr}^{-/-}$mice stimulated by AEW (Figures 7B-D), demonstrating that spinal CXCR3 was indispensable for the anti-pruritic activity of paeonol in AEW-induced dry skin.

\section{The Suppressive Effect of Paeonol on Astrocytic Activation Was Abolished in $\mathrm{CxCr}^{-1-}$ Mice}

Reactive astrocytes in the SDH have been reported in models of dry skin (Green and Dong, 2015; Liu et al., 2016; Tsuda, 2017; Tsuda, 2018) and activated astrocytes may produce CXCR10 to act on neurons through CXCR3 via a paracrine signaling (Jiang et al., 2017; Doron et al., 2019; Petrisko et al., 2020). To explore whether paeonol affects the activation of astrocytes in AEW model, we tested the mRNA levels of toll-like receptor 4 (Tlr4) (Liu et al., 2016) (Figure 8A), lipocalin 2 (Lcn2) (ShiratoriHayashi et al., 2021) (Figure 8B) and other genes in the spinal cord related to reactive astrocytes (Liddelow et al., 2017) (Figures 8C-H), including heat shock protein 1 (Hspb1), CD44 antigen (Cd44), ceruloplasmin (Cp), serine/ cysteine peptidase inhibitor, clade A, member 3N (Serpina3n), vimentin (Vim) and glial fibrillary acidic protein (Gfap), and found that their expression were significantly decreased by paeonol treatment as expected. Conversely, paeonol failed to unregulated the levels of these genes in AEW-induced $\mathrm{CxCr}^{-1-}$ mice (Figures 9A-H). Therefore, paeonol was able to inhibit the astrocytic activation of AEW mice driven by CXCR3, suggesting that targeting spinal CXCR3 is a potential approach for treating the chronic itch with activated astrocytes.

\section{DISCUSSION}

Nowadays, increasing attention has been paid to the antidermatitis activities of paeonol. For example, paeonol inhibits the maturation and activation of DCs by reducing MyD88 and TLR8 proteins in the TLR7/8 signalling pathway, and ultimately alleviates psoriasis-like skin lesions in BALB/c mice (Meng et al., 2017). For UV-induced skin disorders, paeonol ameliorates SUVinduced skin inflammation by inhibiting the increase of TOPK, the phosphorylation of $\mathrm{p} 38$, JNKs and H2AX, and the secretion of IL- 6 and TNF- $\alpha$ in Babl/c mouse. Paeonol also attenuates UVBinduced matrix metalloproteinase- 1 production and promotes procollagen type I in hairless mice to protect skin from UVBinduced photoaging (Xue et al., 2017; Sun et al., 2018). In addition, paeonol improves the development of DNCBinduced $\mathrm{AD}$ in the $\mathrm{BALB} / \mathrm{c}$ mice by reducing severity of the lesions, epidermal thickness and mast cell infiltration, accompanied by the reduction of immunoglobulin $\mathrm{E}$ and inflammatory cytokines, and regulation of the $\mathrm{T}$ helper (Th) cell subset (Th1/Th2) ratio (Meng et al., 2019). Nevertheless, these pharmacological studies mainly focused on anti- 

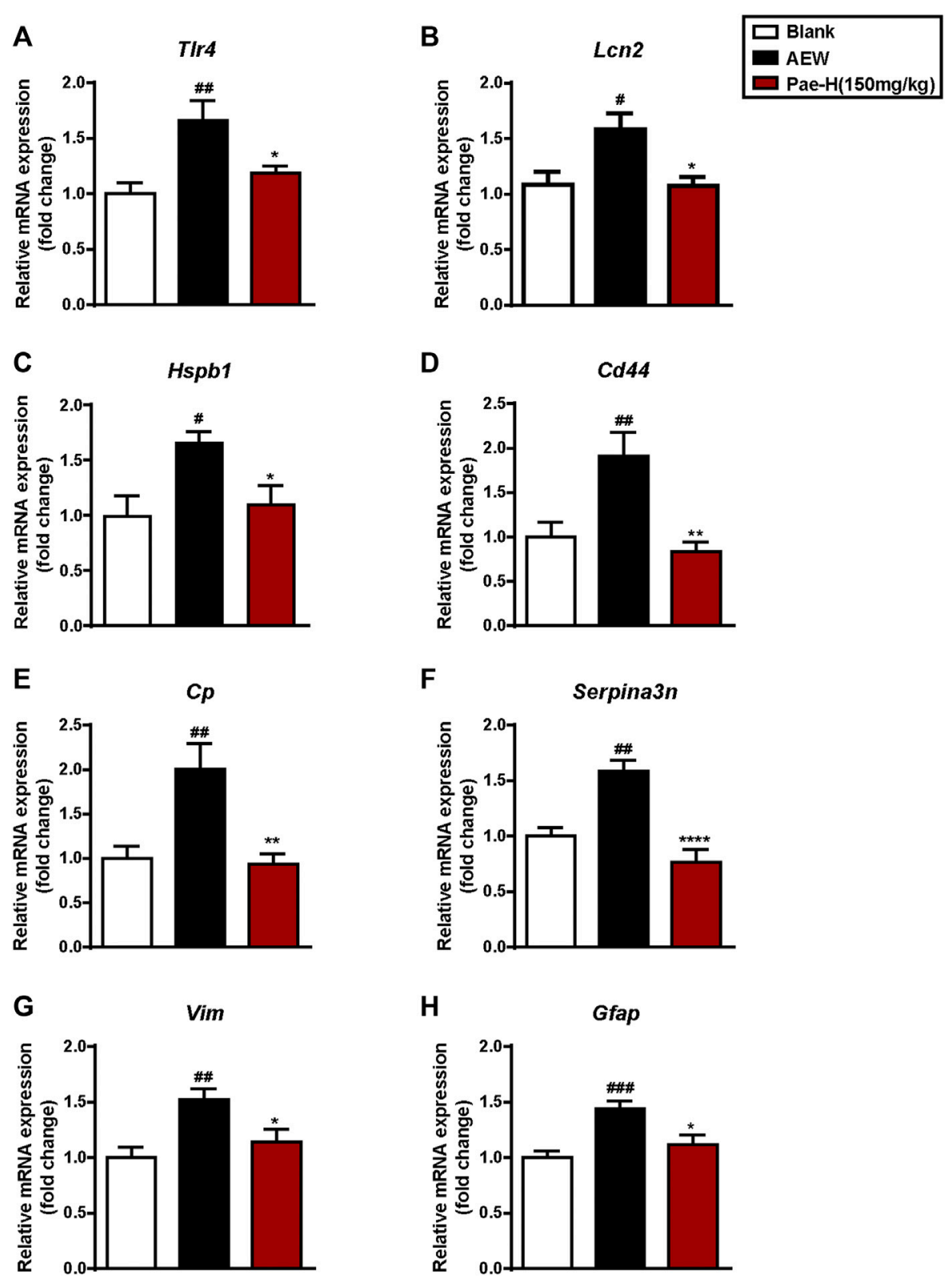

FIGURE 8 | Paeonol suppressed the expression of astrocyte activity-dependent genes in the spinal cord of AEW mice. (A-H) Administration of paeonol inhibited the mRNA levels of genes related to reactive astrocytes ( $n=5-9$ each group). Data were expressed as mean \pm SEM. ${ }^{\#} p<0.05,{ }^{\# \#} p<0.01$, ${ }^{\# \#} p<0.001$, compared to the blank group; ${ }^{*} p<0.05,{ }^{* *} p<0.01,{ }^{* * *} p<0.001$, compared to the AEW model group.

inflammatory activity of paeonol. Although the inhibitory effects of paeonol on scratching behavior and mast cell degranulation in the acute itch model induced by compound 48/80 (Lee et al., 2008) supported our findings in AEW-induced dry skin mice, the mechanism of its ability of antipruritus, especially for chronic itch, is poorly understood so far.

CXCR3 signaling has been reported involving in various human diseases, including chronic inflammation, immune dysfunction, cancer, metastasis, and pruritus (Van Raemdonck et al., 2015). CXCR3/CXCL10 axis contributes to the recruitment of inflammatory cells and the production of cytokines in RA progression (Lee et al., 2017), chronic prostatitis (Hua et al., 2021), inflammatory bowel disease (Zhao et al., 2017), etc. In addtion to its traditional functions in inflammation and immunity as we known, the potential role of CXCR3 in chronic dermatitis on itch sensation has attracted growing attention in recent years. AEW treatment induced the elevated levels of CXCR3 and CXCL10 in the spinal cord, and $\mathrm{CXCr}^{-1-}$ mice showed reduced scratching behavior than that in control mice (Jing et al., 2018). Consistent with previous studies, we investigated that the expression of CXCR3 in the spinal cord of AEW mice was significantly elevated along the period. Treatment 

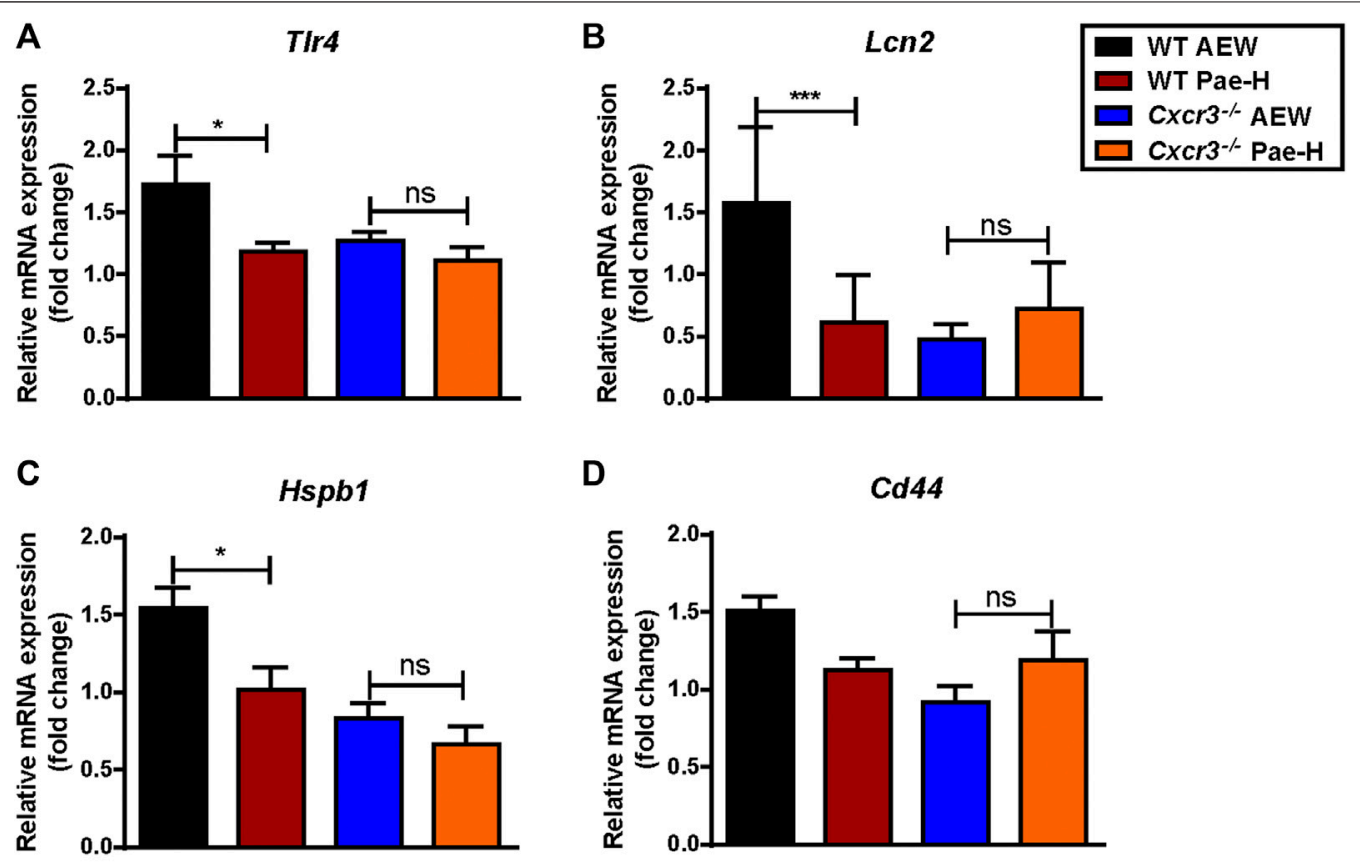

D $\quad$ Cd44
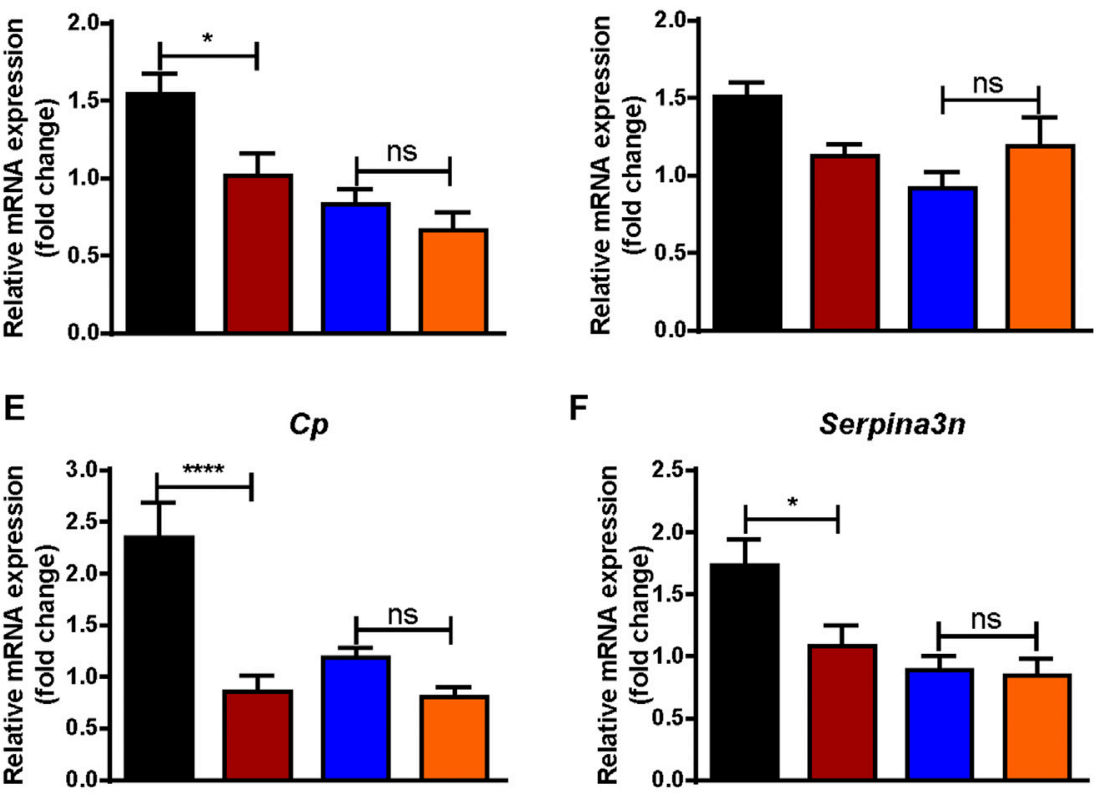

F
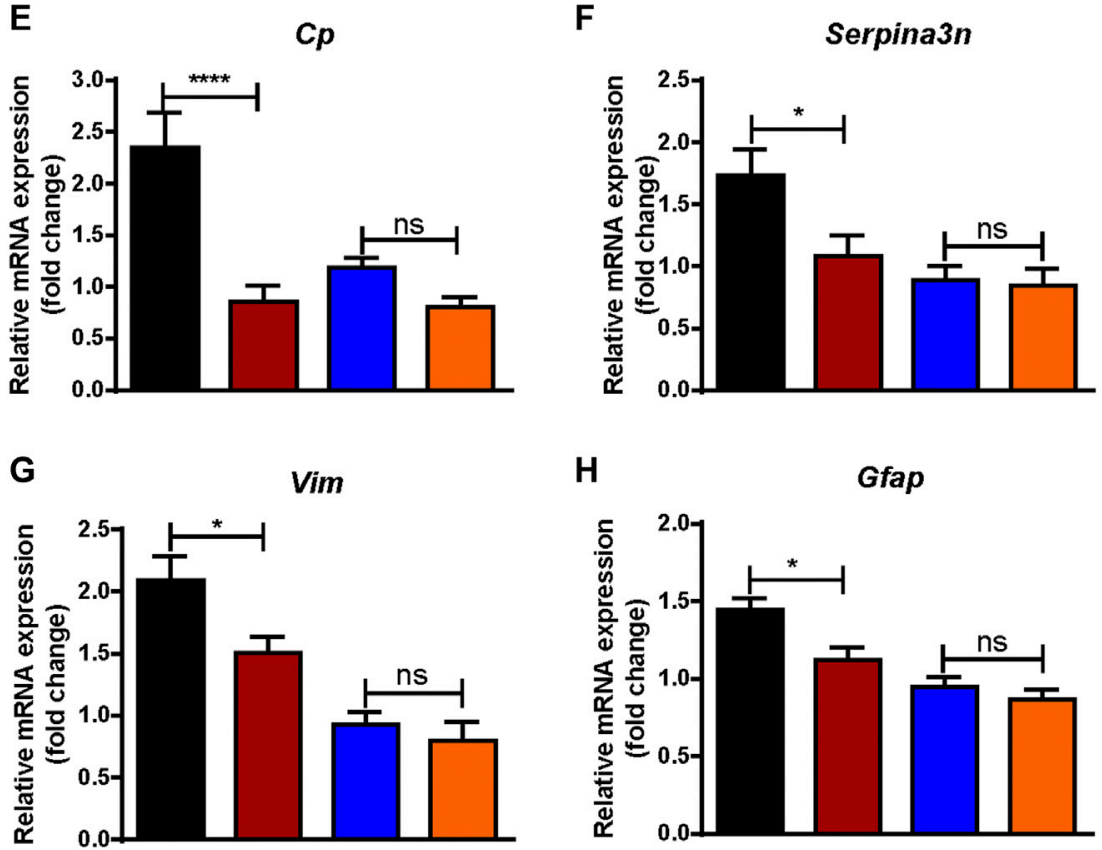

H

Gfap

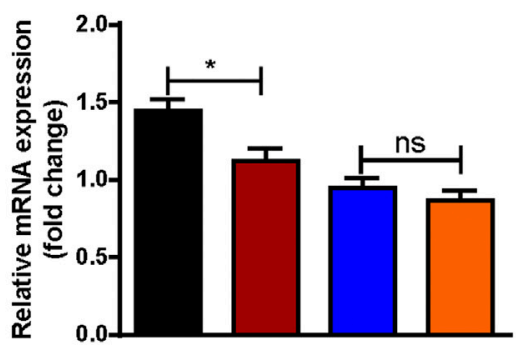

FIGURE 9 | The effects of paeonol on astrocyte activity-dependent genes in WT mice and Cxcr3 ${ }^{-/-}$mice. (A-H) The effects of paeonol on mRNA levels of genes related to reactive astrocytes in WT and $\mathrm{CxCr}^{-/-}$mice $\left(n=5-9\right.$ each group). Data were expressed as mean \pm SEM. ${ }^{*} p<0.05,{ }^{* *} p<0.01,{ }^{* * *} p<0.001,{ }^{* * * *} p<0.0001$, compared to the WT-AEW model group.

of paeonol effectively reversed the expression of spinal CXCR3 at both mRNA and protein levels. Though the expression of spinal Cxcl10 in AEW-induced mice did not increase as previously reported, it was reduced by paeonol significantly as well, supporting the hypothesis that paeonol exerted as an anti- pruritic agent in the treatment of dry skin via inhibiting spinal CXCR3-mediated signalling. In addtion to $C x c r 3 / C x c l 10$ pair, it's worth mentioning that paeonol also significantly down-regulated the mRNA levels of spinal Ccl6, Ccl9 and Ccl17. However, there is little evidence of their exact roles in itch sensation. 
Central circuit mechanisms that contribute to the sensation of itch, including the crucial role of astrocytes in maintaining the development and pathogenesis of chronic itch, has attracted widespread attention (Green and Dong, 2015; Chen and Sun, 2020). Recent studies have proved that the repetitive scratching behaviors in chronic itch models require the contribution of astrocytic molecules including the transcription factor signal transducer and activator of transcription 3 (STAT3) and the receptor toll-like receptor 4 (TLR4) (Tsuda, 2018). Behavioral evidence using NC/Nga mice suggested that STAT3-dependent spinal cord central sensitization occurs via amplification of GRP signalling under chronic itch conditions (Shiratori-Hayashi et al., 2015). LCN2 upregulated in STAT3-dependent reactive astrocytes in the SDH amplifies GRP signalling and contributes to the progression and maintenance of chronic itch (Shiratori-Hayashi et al., 2015; Shiratori-Hayashi and Tsuda, 2020; Shiratori-Hayashi et al., 2021). Spinal TLR4 is necessary for AEW-induced chronic itch in the cheek model. AEW induces persistent upregulation of Tlr4 mRNA in GFAPexpressing astrocytes and TLR4-dependent astrogliosis (GFAP upregulation) in the spinal dorsal horn (Tsuda, 2018). In addition, reactive astrogliosis consists of a rapid, but quickly weakened, induction of gene expression after injury, including $C P$ (Ryan et al., 2018; Wu et al., 2018), Vim (Qian et al., 2015; Smith et al., 2018; Adolf et al., 2019) and Serpina3n (Zamanian et al., 2012; Domowicz et al., 2021; Ji et al., 2021). In our study, their expression responds to AEW stimulation increased significantly.

In addition, chemokines expressed in the central nervous system also participate in the pathogenesis of pain and itch via neuron-glia interaction in the spinal cord. CXCL13 is highly upregulated in spinal neurons after spinal nerve ligation and induces spinal astrocyte activation via receptor CXCR5 (Zhang et al., 2017; Liu S. et al., 2019). Absence of the chemokine receptor CXCR3 inhibited both astrocytic activation and chronic itch in AEW-induced dry skin model mice (Jing et al., 2018; ShiratoriHayashi and Tsuda, 2020). In this research, the expression of astrocyte activation-dependent genes (e.g., Tlr4, Lcn2, Hspb1 and Cd44) were significantly decreased by paeonol administration (Figure 4), proving that paeonol could inhibit AEW-induced activation of astrocytes in the spinal cord. However, the inhibitory effects of paeonol on these genes was abolished in CXCR3-deficient mice (Figure 5), supporting that paeonol relieved the pruritus of dry skin mice by inhibiting the spinal astrocytic activation driven by CXCR3. In fact, in recent years, the research field has made substantial progress in understanding how astrocytes at the spinal cord level participate in the regulation of chronic pain and pruritus through neuron-glia and glial-glia interactions. In addition to the effects of cytokines and chemokines in causing skin itching in the periphery, they may also participate in the abovementioned interactions through the activation of astrocytes, causing chronic itching. It has been clearly showed that the mediators produced by astrocytes such as cytokines and chemokines are powerful neuromodulators that can regulate the itch circuit and cause central sensitization. For further studies, we plan to focus on the exact process of neuronal CXCR3 mediates the astrogliosis in the $\mathrm{SDH}$, and the role of paeonol plays in it.

In conclusion, for the first time we investigated that paeonol could ameliorate both the chronic itch and skin inflammation of AEW-induced dry skin in mice. It exerted good anti-pruritic effect through suppressing the astrocyte activation which was driven by CXCR3 in the spinal cord. Thus, searching for new natural compounds that target spinal CXCR3 signaling may provide novel therapeutic potential for the treatment of dry skin disease.

\section{DATA AVAILABILITY STATEMENT}

The original contributions presented in the study are included in the article/supplementary material, further inquiries can be directed to the corresponding authors.

\section{ETHICS STATEMENT}

The animal study was reviewed and approved by the Animal Ethics Committee of South-Central University for Nationalities.

\section{AUTHOR CONTRIBUTIONS}

Conceptualization: ZM and HX; Methodology: WW and ZZ; Investigation: QL, YL, and WW; Writing-Original Draft: QL; Writing-Review and Editing: WW and ZZ; Supervision: ZM; Funding Acquisition: ZM.

\section{FUNDING}

This work is supported by the National Major Scientific and Technological Special Project for "Significant New Drugs Development" of China (Grant No. 2017ZX09301060), the Major Scientific and Technological Special Project of Hubei Province (Grant No. 2020ACA019) and the Special Fund for the Basic Scientific Research of Central Colleges, South-Central University for Nationalities (Grant No. CZJ19001).

\section{ACKNOWLEDGMENTS}

The authors thank Dr. Yongjing Gao at Nantong University in China for the breeders of $\mathrm{Cxcr}^{-/-}$mice used in this project. 


\section{REFERENCES}

Adki, K. M., and Kulkarni, Y. A. (2020). Chemistry, Pharmacokinetics, Pharmacology and Recent Novel Drug Delivery Systems of Paeonol. Life Sci. 250, 117544. doi:10.1016/j.lfs.2020.117544

Adki, K. M., and Kulkarni, Y. A. (2021). Neuroprotective Effect of Paeonol in Streptozotocin-Induced Diabetes in Rats. Life Sci. 271, 119202. doi:10.1016/ j.lfs.2021.119202

Adolf, A., Rohrbeck, A., Münster-Wandowski, A., Johansson, M., Kuhn, H. G., Kopp, M. A., et al. (2019). Release of Astroglial Vimentin by Extracellular Vesicles: Modulation of Binding and Internalization of C3 Transferase in Astrocytes and Neurons. Glia 67, 703-717. doi:10.1002/glia.23566

Chen, X. J., and Sun, Y. G. (2020). Central Circuit Mechanisms of Itch. Nat. Commun. 11, 3052. doi:10.1038/s41467-020-16859-5

Domowicz, M. S., Chan, W. C., Claudio-Vázquez, P., Gonzalez, T., and Schwartz, N. B. (2021). Brain Transcriptome Analysis of a CLN2 Mouse Model as a Function of Disease Progression. J. Neuroinflamm. 18, 262. doi:10.1186/s12974021-02302-z

Dong, X., and Dong, X. (2018). Peripheral and Central Mechanisms of Itch. Neuron 98, 482-494. doi:10.1016/j.neuron.2018.03.023

Doron, H., Amer, M., Ershaid, N., Blazquez, R., Shani, O., Lahav, T. G., et al. (2019). Inflammatory Activation of Astrocytes Facilitates Melanoma Brain Tropism via the CXCL10-CXCR3 Signaling Axis. Cel. Rep. 28, 1785-1798.e6. doi:10.1016/ j.celrep.2019.07.033

Feng, J., Yang, P., Mack, M. R., Dryn, D., Luo, J., Gong, X., et al. (2017). Sensory TRP Channels Contribute Differentially to Skin Inflammation and Persistent Itch. Nat. Commun. 8, 980. doi:10.1038/s41467-017-01056-8

Fernandes, E. S., Vong, C. T., Quek, S., Cheong, J., Awal, S., Gentry, C., et al. (2013). Superoxide Generation and Leukocyte Accumulation: Key Elements in the Mediation of Leukotriene $\mathrm{B}_{4}$-induced Itch by Transient Receptor Potential Ankyrin 1 and Transient Receptor Potential Vanilloid 1. FASEB J. 27, 1664-1673. doi:10.1096/fj.12-221218

Gao, L., Wang, Z., Lu, D., Huang, J., Liu, J., and Hong, L. (2019). Paeonol Induces Cytoprotective Autophagy via Blocking the Akt/mTOR Pathway in Ovarian Cancer Cells. Cel. Death Dis. 10, 609. doi:10.1038/s41419-019-1849-x

Green, D., and Dong, X. (2015). Supporting Itch: a New Role for Astrocytes in Chronic Itch. Nat. Med. 21, 841-842. doi:10.1038/nm.3921

Harada, M., and Yamashita, A. (1969). Pharmacological Studies on the Root Bark of Paeonia Moutan. I. Central Effects of Paeonol. Yakugaku Zasshi 89, 1205-1211. doi:10.1248/yakushi1947.89.9_1205

Hua, X., Ge, S., Zhang, M., Mo, F., Zhang, L., Zhang, J., et al. (2021). Pathogenic Roles of CXCL10 in Experimental Autoimmune Prostatitis by Modulating Macrophage Chemotaxis and Cytokine Secretion. Front. Immunol. 12, 706027. doi:10.3389/fimmu.2021.706027

Ji, C., Tang, Y., Zhang, Y., Li, C., Liang, H., Ding, L., et al. (2022). Microglial Glutaminase 1 Deficiency Mitigates Neuroinflammation Associated Depression. Brain Behav. Immun. 99, 231-245. doi:10.1016/j.bbi.2021.10.009

Jiang, B. C., He, L. N., Wu, X. B., Shi, H., Zhang, W. W., Zhang, Z. J., et al. (2017). Promoted Interaction of $\mathrm{C} / \mathrm{EBPa}$ with Demethylated Cxcr3 Gene Promoter Contributes to Neuropathic Pain in Mice. J. Neurosci. 37, 685-700. doi:10.1523/ JNEUROSCI.2262-16.2016

Jiang, H., Cui, H., Wang, T., Shimada, S. G., Sun, R., Tan, Z., et al. (2019). CCL2/ CCR2 Signaling Elicits Itch- and Pain-like Behavior in a Murine Model of Allergic Contact Dermatitis. Brain Behav. Immun. 80, 464-473. doi:10.1016/ j.bbi.2019.04.026

Jiang, B. C., Liu, T., and Gao, Y. J. (2020). Chemokines in Chronic Pain: Cellular and Molecular Mechanisms and Therapeutic Potential. Pharmacol. Ther. 212, 107581. doi:10.1016/j.pharmthera.2020.107581

Jin, X., Wang, J., Xia, Z. M., Shang, C. H., Chao, Q. L., Liu, Y. R., et al. (2016). Antiinflammatory and Anti-oxidative Activities of Paeonol and its Metabolites through Blocking MAPK/ERK/p38 Signaling Pathway. Inflammation 39, 434-446. doi:10.1007/s10753-015-0265-3

Jing, P. B., Cao, D. L., Li, S. S., Zhu, M., Bai, X. Q., Wu, X. B., et al. (2018). Chemokine Receptor CXCR3 in the Spinal Cord Contributes to Chronic Itch in Mice. Neurosci. Bull. 34, 54-63. doi:10.1007/s12264017-0128-z
Koga, K., Yamagata, R., Kohno, K., Yamane, T., Shiratori-Hayashi, M., Kohro, Y., et al. (2020). Sensitization of Spinal Itch Transmission Neurons in a Mouse Model of Chronic Itch Requires an Astrocytic Factor. J. Allergy Clin. Immunol. 145, 183-191.e10. doi:10.1016/j.jaci.2019.09.034

Lee, B., Shin, Y. W., Bae, E. A., Han, S. J., Kim, J. S., Kang, S. S., et al. (2008), Antiallergic Effect of the Root of Paeonia Lactiflora and its Constituents Paeoniflorin and Paeonol. Arch. Pharm. Res. 31, 445-450. doi:10.1007/ s12272-001-1177-6

Lee, J. H., Kim, B., Jin, W. J., Kim, H. H., Ha, H., and Lee, Z. H. (2017). Pathogenic Roles of CXCL10 Signaling through CXCR3 and TLR4 in Macrophages and T Cells: Relevance for Arthritis. Arthritis Res. Ther. 19, 163. doi:10.1186/ s13075-017-1353-6

Lichterfeld-Kottner, A., El Genedy, M., Lahmann, N., Blume-Peytavi, U., Büscher, A., and Kottner, J. (2020). Maintaining Skin Integrity in the Aged: A Systematic Review. Int. J. Nurs. Stud. 103, 103509. doi:10.1016/ j.ijnurstu.2019.103509

Liddelow, S. A., Guttenplan, K. A., Clarke, L. E., Bennett, F. C., Bohlen, C. J., Schirmer, L., et al. (2017). Neurotoxic Reactive Astrocytes are Induced by Activated Microglia. Nature 541 (7638), 481-487. doi:10.1038/nature21029

Liu, B. W., Li, Z. X., He, Z. G., Wang, Q., Liu, C., Zhang, X. W., et al. (2019). Altered Expression of Itch-Related Mediators in the Lower Cervical Spinal Cord in Mouse Models of Two Types of Chronic Itch. Int. J. Mol. Med. 44, 835-846. doi:10.3892/ijmm.2019.4253

Liu, T., Han, Q., Chen, G., Huang, Y., Zhao, L. X., Berta, T., et al. (2016). Toll-like Receptor 4 Contributes to Chronic Itch, Alloknesis, and Spinal Astrocyte Activation in Male Mice. Pain 157, 806-817. doi:10.1097/ j.pain. 0000000000000439

Liu, C. M., Yang, H. X., Ma, J. Q., Yang, W., Feng, Z. J., Sun, J. M., et al. (2018). Role of AMPK Pathway in lead-induced Endoplasmic Reticulum Stress in Kidney and in Paeonol-Induced protection in Mice. Food Chem. Toxicol. 122, 87-94. doi:10.1016/j.fct.2018.10.024

Liu, S., Liu, X., Xiong, H., Wang, W., Liu, Y., Yin, L., et al. (2019). CXCL13/CXCR5 Signaling Contributes to Diabetes-Induced Tactile Allodynia via Activating pERK, pSTAT3, pAKT Pathways and Pro-inflammatory Cytokines Production in the Spinal Cord of Male Mice. Brain Behav. Immun. 80, 711-724. doi:10.1016/j.bbi.2019.05.020

Meng, Y., Wang, M., Xie, X., Di, T., Zhao, J., Lin, Y., et al. (2017). Paeonol Ameliorates Imiquimod-Induced Psoriasis-like Skin Lesions in BALB/c Mice by Inhibiting the Maturation and Activation of Dendritic Cells. Int. J. Mol. Med. 39, 1101-1110. doi:10.3892/ijmm.2017.2930

Meng, Y., Liu, Z., Zhai, C., Di, T., Zhang, L., Zhang, L., et al. (2019). Paeonol Inhibits the Development of 1-Chloro-2,4-Dinitrobenzene-Induced Atopic Dermatitis via Mast and T Cells in BALB/c Mice. Mol. Med. Rep. 19, 3217-3229. doi:10.3892/mmr.2019.9985

Moniaga, C. S., Tominaga, M., and Takamori, K. (2020). Mechanisms and Management of Itch in Dry Skin. Acta Derm Venereol. 100, adv00024. doi:10.2340/00015555-3344

Oh, M. H., Oh, S. Y., Lu, J., Lou, H., Myers, A. C., Zhu, Z., et al. (2013). TRPA1Dependent Pruritus in IL-13-induced Chronic Atopic Dermatitis. J. Immunol. 191, 5371-5382. doi:10.4049/jimmunol.1300300

Patricio, E. S., Costa, R., Figueiredo, C. P., Gers-Barlag, K., Bicca, M. A., Manjavachi, M. N., et al. (2015). Mechanisms Underlying the Scratching Behavior Induced by the Activation of Proteinase-Activated Receptor-4 in Mice. J. Invest. Dermatol. 135, 2484-2491. doi:10.1038/jid.2015.183

Petrisko, T. J., Bloemer, J., Pinky, P. D., Srinivas, S., Heslin, R. T., Du, Y., et al. (2020). Neuronal CXCL10/CXCR3 Axis Mediates the Induction of Cerebral Hyperexcitability by Peripheral Viral Challenge. Front. Neurosci. 14, 220. doi:10.3389/fnins.2020.00220

Qian, B. J., You, L., Shang, F. F., Liu, J., Dai, P., Lin, N., et al. (2015). Vimentin Regulates Neuroplasticity in Transected Spinal Cord Rats Associated with micRNA138. Mol. Neurobiol. 51, 437-447. doi:10.1007/s12035-014-8745-2

Qu, L., Fu, K., Yang, J., Shimada, S. G., and LaMotte, R. H. (2015). CXCR3 Chemokine Receptor Signaling Mediates Itch in Experimental Allergic Contact Dermatitis. Pain 156, 1737-1746. doi:10.1097/j.pain.0000000000000208

Qu, L., Fu, K., Shimada, S. G., and LaMotte, R. H. (2017). $\mathrm{Cl}^{-}$Channel Is Required for CXCL10-Induced Neuronal Activation and Itch Response in a Murine Model of Allergic Contact Dermatitis. J. Neurophysiol. 118, 619-624. doi:10.1152/jn.00187.2017 
Ryan, F., Zarruk, J. G., Lößlein, L., and David, S. (2018). Ceruloplasmin Plays a Neuroprotective Role in Cerebral Ischemia. Front. Neurosci. 12, 988. doi:10.3389/fnins.2018.00988

Shiratori-Hayashi, M., and Tsuda, M. (2020). Role of Reactive Astrocytes in the Spinal Dorsal Horn under Chronic Itch Conditions. J. Pharmacol. Sci. 144, 147-150. doi:10.1016/j.jphs.2020.07.010

Shiratori-Hayashi, M., Koga, K., Tozaki-Saitoh, H., Kohro, Y., Toyonaga, H., Yamaguchi, C., et al. (2015). STAT3-Dependent Reactive Astrogliosis in the Spinal Dorsal Horn Underlies Chronic Itch. Nat. Med. 21, 927-931. doi:10.1038/nm.3912

Shiratori-Hayashi, M., Yamaguchi, C., Eguchi, K., Shiraishi, Y., Kohno, K., Mikoshiba, K., et al. (2021). Astrocytic STAT 3 Activation and Chronic Itch Require $\mathrm{IP}_{3} \mathrm{R} 1 / \mathrm{TRPC}$-dependent $\mathrm{Ca}^{2+}$ Signals in Mice. J. Allergy Clin. Immunol. 147, 1341-1353. doi:10.1016/j.jaci.2020.06.039

Smith, J. A., Braga, A., Verheyen, J., Basilico, S., Bandiera, S., Alfaro-Cervello, C., et al. (2018). RNA Nanotherapeutics for the Amelioration of Astroglial Reactivity. Mol. Ther. Nucleic Acids 10, 103-121. doi:10.1016/j.omtn.2017.11.008

Su, W., Yu, J., Liu, Q., Ma, L., and Huang, Y. (2020). CXCL12/CXCR4 Signaling Induced Itch and Pain Sensation in a Murine Model of Allergic Contact Dermatitis. Mol. Pain 16, 1744806920926426. doi:10.1177/1744806920926426

Sun, Y. G., Zhao, Z. Q., Meng, X. L., Yin, J., Liu, X. Y., and Chen, Z. F. (2009). Cellular Basis of Itch Sensation. Science 325, 1531-1534. doi:10.1126/ science. 1174868

Sun, Z., Du, J., Hwang, E., and Yi, T. H. (2018). Paeonol Extracted from Paeonia Suffruticosa Andr. Ameliorated UVB-Induced Skin Photoaging via DLD/Nrf2/ARE and MAPK/AP-1 Pathway. Phytother Res. 32, 1741-1749. doi:10.1002/ptr.6100

Tsuda, M. (2017). Spinal Dorsal Horn Astrocytes: New Players in Chronic Itch. Allergol. Int. 66, 31-35. doi:10.1016/j.alit.2016.07.007

Tsuda, M. (2018). Astrocytes in the Spinal Dorsal Horn and Chronic Itch. Neurosci. Res. 126, 9-14. doi:10.1016/j.neures.2017.08.010

Van Raemdonck, K., Van den Steen, P. E., Liekens, S., Van Damme, J., and Struyf, S. (2015). CXCR3 Ligands in Disease and Therapy. Cytokine Growth Factor. Rev. 26, 311-327. doi:10.1016/j.cytogfr.2014.11.009

Wang, W., Wang, H., Zhao, Z., Huang, X., Xiong, H., and Mei, Z. (2020). Thymol Activates TRPM8-Mediated $\mathrm{Ca}^{2+}$ Influx for its Antipruritic Effects and Alleviates Inflammatory Response in Imiquimod-Induced Mice. Toxicol. Appl. Pharmacol. 407, 115247. doi:10.1016/j.taap.2020.115247

White-Chu, E. F., and Reddy, M. (2011). Dry Skin in the Elderly: Complexities of a Common Problem. Clin. Dermatol. 29, 37-42. doi:10.1016/j.clindermatol.2010.07.005

Wilson, S. R., Nelson, A. M., Batia, L., Morita, T., Estandian, D., Owens, D. M., et al. (2013). The Ion Channel TRPA1 Is Required for Chronic Itch. J. Neurosci. 33, 9283-9294. doi:10.1523/JNEUROSCI.5318-12.2013

Wilzopolski, J., Kietzmann, M., Mishra, S. K., Stark, H., Bäumer, W., and Rossbach, K. (2021). TRPV1 and TRPA1 Channels Are Both Involved Downstream of Histamine-Induced Itch. Biomolecules 11, 1166. doi:10.3390/biom11081166

Wu, Y., Shen, L., Wang, R., Tang, J., Ding, S. Q., Wang, S. N., et al. (2018). Increased Ceruloplasmin Expression Caused by Infiltrated Leukocytes, Activated Microglia, and Astrocytes in Injured Female Rat Spinal Cords. J. Neurosci. Res. 96, 1265-1276. doi:10.1002/jnr.24221

Xiang, X., Tu, C., Li, Q., Wang, W., Huang, X., Zhao, Z., et al. (2020). Oxymatrine Ameliorates Imiquimod-Induced Psoriasis Pruritus and Inflammation through
Inhibiting Heat Shock Protein 90 and Heat Shock Protein 60 Expression in Keratinocytes. Toxicol. Appl. Pharmacol. 405, 115209. doi:10.1016/ j.taap.2020.115209

Xiao, W. M., Zha, X. S., and Wang, Y. F. (2020). Effects of Paeonol on Expressions of SP, NK1R and Mast Cell Activation in Eczema Model Mice. Tianjin Med. J. 48, 09. doi:10.11958/20200896

Xu, J., Xiong, H., Zhao, Z., Luo, M., Ju, Y., Yang, G., et al. (2021). Genistein Suppresses Allergic Contact Dermatitis through Regulating the MAP2K2/ERK Pathway. Food Funct. 12, 4556-4569. doi:10.1039/d0fo03238g

Xue, P., Wang, Y., Zeng, F., Xiu, R., Chen, J., Guo, J., et al. (2017). Paeonol Suppresses Solar Ultraviolet-Induced Skin Inflammation by Targeting T-LAK Cell-Originated Protein Kinase. Oncotarget 8, 27093-27104. doi:10.18632/ oncotarget.15636

Zamanian, J. L., Xu, L., Foo, L. C., Nouri, N., Zhou, L., Giffard, R. G., et al. (2012). Genomic Analysis of Reactive Astrogliosis. J. Neurosci. 32, 6391-6410. doi:10.1523/JNEUROSCI.6221-11.2012

Zhang, Z. J., Jiang, B. C., and Gao, Y. J. (2017). Chemokines in Neuron-Glial Cell Interaction and Pathogenesis of Neuropathic Pain. Cell Mol Life Sci 74, 3275-3291. doi:10.1007/s00018-017-2513-1

Zhang, L., Li, D. C., and Liu, L. F. (2019). Paeonol: Pharmacological Effects and Mechanisms of Action. Int. Immunopharmacol 72, 413-421. doi:10.1016/ j.intimp.2019.04.033

Zhang, L., Chen, W. X., Li, L. L., Cao, Y. Z., Geng, Y. D., Feng, X. J., et al. (2020). Paeonol Suppresses Proliferation and Motility of Non-Small-Cell Lung Cancer Cells by Disrupting STAT3/NF-Kb Signaling. Front. Pharmacol. 11, 572616. doi:10.3389/fphar.2020.572616

Zhao, Z. Q., Huo, F. Q., Jeffry, J., Hampton, L., Demehri, S., Kim, S., et al. (2013). Chronic Itch Development in Sensory Neurons Requires BRAF Signaling Pathways. J. Clin. Invest. 123, 4769-4780. doi:10.1172/JCI70528

Zhao, Q., Kim, T., Pang, J., Sun, W., Yang, X., Wang, J., et al. (2017). A Novel Function of CXCL10 in Mediating Monocyte Production of Proinflammatory Cytokines. J. Leukoc. Biol. 102, 1271-1280. doi:10.1189/jlb.5A0717-302

Conflict of Interest: The authors declare that the research was conducted in the absence of any commercial or financial relationships that could be construed as a potential conflict of interest.

Publisher's Note: All claims expressed in this article are solely those of the authors and do not necessarily represent those of their affiliated organizations, or those of the publisher, the editors and the reviewers. Any product that may be evaluated in this article, or claim that may be made by its manufacturer, is not guaranteed or endorsed by the publisher.

Copyright (C) 2022 Wang, Li, Zhao, Liu, Wang, Xiong and Mei. This is an open-access article distributed under the terms of the Creative Commons Attribution License (CC $B Y)$. The use, distribution or reproduction in other forums is permitted, provided the original author(s) and the copyright owner(s) are credited and that the original publication in this journal is cited, in accordance with accepted academic practice. No use, distribution or reproduction is permitted which does not comply with these terms. 